\title{
EXISTENCE THEOREMS FOR IMPLICIT FUNCTIONS OF REAL VARIABLES*
}

\section{BY H. J. ETTLINGER}

The classical theorems on implicit functions make use of the continuity of the given functions and their partial derivatives when all the variables are considered as independent. $\dagger$ The existence theorems established herein bring the implicit function theorems into line with the most recent developments of real variable theory. $\ddagger$ Two of my students, W. M. Whyburn and J. H. Sturdivant, have made use of Theorem I in connection with studies of the properties of solutions of ordinary linear differential equations with summable coefficients.

In Theorem I sufficient conditions are given to ensure a single-valued continuous solution $y=y(x)$ of the relation $F(x, y)=0$. These conditions reduce the classical conditions considerably.

By introducing symmetry in $x$ and $y$ save in the final two hypotheses of Theorem II, sufficient conditions are given to ensure a single-valued absolutely continuous solution (with a summable derivative almost everywhere).

* Presented to the Society, September 8, 1926.

† See Goursat-Hedrick, Mathematical Analysis, vol. 1, 1904, Chapter II p. $35 \mathrm{ff}$. For a summary of the results and references to original sources, see Bliss, Princeton Colloquium Lectures, Fundamental Existence Theorems, delivered in 1909, published by the Society in 1913, New York.

A distinct lightening of the classical conditions for the existence of implicit functions is to be found in a paper by Hedrick and Westfall, Bulletin de la Société de France, vol. 44 (1916), pp. 1-14.

¥ See Carathéodory, Vorlesungen über reelle Funktionen, Leipzig, Teubner,1918. Very recently (August, 1927) a second edition of this treatise has appeared with only a few additional references to more recent literature. The page references given in later footnotes to the present work apply equally well to the second (1927) edition. 
Theorem I. Hypothesis. 1. $D_{h}$ is a neighborhood of a point $(X, Y)$ in the $(x, y)$ plane, $|x-X| \leqq h,|y-Y| \leqq h, h>0$. 2. $F(x, y)$ is defined in $D_{h}$ and vanishes at $(X, Y)$. 3. (a) $F(x, y)$ is absolutely continuous in $y$ on $|x-X| \leqq h$, for every fixed $x$ on $|x-X| \leqq h ;(b)\left|F_{y}^{\prime}(x, y)\right| \leqq M(y)$ for all values of $x$ on $|x-X| \leqq h$, where $M(y)$ is summable in $y$ on $|y-Y| \leqq h$; (c) $F_{y}^{\prime}(x, y)$ is continuous in $x$ for every fixed $y$ almost everywhere on $|y-Y| \leqq h .4 . F(x, Y)$ is continuous in $x$ on $|x-X|$ $\leqq h$. 5. $F_{y}^{\prime}(x, y)>0(<0)^{*}$ for each fixed $x$ on $|x-X| \leqq h$, almost everywhere on $|y-Y| \leqq h$.

Conclusion. There exists in $D_{k}, 0<k \leqq h$, a unique single-valued continuous function $y=y(x)$, such that we have 1. $F(x, y(x)) \equiv 0$ in $D_{k}, 2 . \quad Y=y(X), 3 . y(x)$ is continuous on $|x-X| \leqq k$.

Proof. By a theorem due to Carathéodory $†$ it follows that $F(x, y)$ is continuous in $(x, y)$ at $(x, Y)$ for $|x-X| \leqq h$ and

$$
F(x, y)=F(x, Y)+\int_{Y}^{y} F_{t}^{\prime}(x, t) d t .
$$

From hypothesis 5 , it follows that for a fixed $x, F(x, y)$ is a monotonic increasing (decreasing) function of $y$ on $|y-Y| \leqq h$, and since $F(X, Y)=0$,

$$
F(X, y)=\int_{Y}^{y} F_{t}^{\prime}(X, t) d t .
$$

Hence $F(X, y)<0$ for $y<Y$, and $F(X, y)>0$ for $y>Y$, or $F(X, Y-h)<0$ and $F(X, Y+h)>0$.

Since $F(x, y)$ is continuous in $x$, it follows that there exists a neighborhood of $(X, Y)$ contained in $D_{h}$ such that $F(x, Y-k)<0$ and $F(x, Y+k)>0$ for every $x$ on $|x-X| \leqq k$.

* For the case $(<0)$ all the subsequent inequality signs are reversed.

$\dagger$ Loc. cit., p. 678, Satz 5. The theorem made use of here is not explicitly stated by Caratheodory, but is implicit in the existence theorem for differential equations cited above. See my note, On continuity in several variables, this Bulletin, vol. 33 (1927), p. 37. Hypotheses $3(a)$ and $3(b)$ of Theorem I above should replace hypotheses (2) and (3) of the theorem of my note. 
But for any fixed $x$ on $|x-X| \leqq k, F(x, y)$ is monotonic increasing in $y$. Hence for each $x$ on $|x-X| \leqq k$, there is one and only one value of $y, y=y(x)$, on $|y-Y| \leqq k$, such that $F(x), y(x) \equiv 0$. Now for $x=X$, we see that the corresponding value of $y$ is $Y$. Finally by the very method* of obtaining $y(x)$, we see that $y(x)$ is continuous in $x$ on $|x-X| \leqq k$.

Theorem II. Hypothesis. 1, 2, 3, 5 remain as in Theorem I. 4.(a) $F(x, y)$ is absolutely continuous $\dagger$ in $x$ on $|x-X| \leqq h$, for every fixed $y$ on $|y-Y| \leqq h ;(b)\left|F_{x}^{\prime}(x, y)\right|$ $\leqq N(x)$ for all values of $y$ on $|y-Y| \leqq h$, where $N(x)$ is summable in $x$ on $|x-X| \leqq h ;(c) F_{x}^{\prime}(x, y)$ is continuous in $y$ for every fixed $x$ almost everywhere on $|x-X| \leqq h$. 6. $\left|F_{x}^{\prime}(x, y) / F_{y}^{\prime}(x, y)\right| \leqq K(x)$ for every $y$ on $|y-Y| \leqq h$, where $K(x)$ is summable in $x$ on $|x-X| \leqq h$.

Conclusion. 1. There is one and only one solution of Theorem I, $y=y(x)$, which is absolutely continuous in $x$ on $|x-X| \leqq k$, and 2. $y_{x}^{\prime}=-F_{x}^{\prime}(x, y) / F_{y}^{\prime}(x, y)$ almost everywhere on $|x-X| \leqq k$, where $y_{x}^{\prime}$ is summable on $|x-X| \leqq k$.

Proof. Condition 4 of Theorem II carries with it condition 4 of Theorem I. From Theorem I we have a solution $y=y(x)$, such that $F(x, y(x)) \equiv 0$. Let $(x, y)$ and $(x+\Delta x, y+\Delta y)$ be any two points on $F(x, y(x)) \equiv 0$. We may write the identity

$$
\frac{\Delta y}{\Delta x}=-\frac{\int_{x}^{x+\Delta x} F_{t}^{\prime}(t, y+\Delta y) d t}{\frac{\int_{y}^{y+\Delta y} F_{t}^{\prime}(x, t) d t}{\Delta y}}
$$

* This follows exactly as in the classical theorem, see Goursat-Hedrick, loc. cit., p. 37 . and $y$.

$\dagger$ Hypothesis 4 is exactly symmetric to hypothesis 3 with respect to $x$ 
The right side of (1) has the limit*

$$
-\frac{F_{x}^{\prime}(x, y(x))}{F_{y}^{\prime}(x, y(x))}
$$

except for a null set of $x$ values on $|x-X| \leqq k$. Hence the left side of (1) approaches a limit almost everywhere on $|x-X| \leqq k$, or

$$
y_{x}^{\prime}=-\frac{F_{x}^{\prime}(x, y(x))}{F_{y}^{\prime}(x, y(x))} .
$$

By a theorem due to Caratheodory $\dagger$ the numerator and denominator of (2) are measurable functions of $x$ on $|x-X|$ $\leqq k$, and hence summable by hypothesis 6 . Hence by (3) $y(x)$ is absolutely continuous in $x$ on $|x-X| \leqq k$.

The above theorems may be extended by the usual method of induction to a system of $n$ functions in $n$ dependent variables and $m$ independent variables.

The University of Texas

* This follows from a generalization of Theorem III of my review, Schlesinger on Lebesgue integrals, this Bulletin, vol. 33 (1927) p. 111. A detailed proof is given by W. M. Whyburn in his dissertation as yet unpublished (offered to the Transactions of this Society).

$\dagger$ Loc. cit., p. 665, Satz 1.

‡ See Goursat-Hedrick, loc. cit., p. 45 , etc. 